\title{
Influence of Primary Structure on Fragmentation of Native-Like Proteins by \\ Ultraviolet Photodissociation
}

Luis A. Macias, Sarah N. Sipe, Inês C. Santos, Aarti Bashyal, M. Rachel Mehaffey, Jennifer S. Brodbelt*

Department of Chemistry, The University of Texas at Austin, Austin, TX, 78712

*to whom correspondence may be addressed: ibrodbelt@cm.utexas.edu

\begin{tabular}{|c|c|c|}
\hline & Content & Page no. \\
\hline 1 & Table S1. Fragment ions in UVPD dataset. & Excel Sheet \\
\hline 2 & Table S2. Proteins and protein complexes in UVPD dataset. & S-2 \\
\hline 3 & Table S3. Number of assigned fragment ions per type. & S-3 \\
\hline 4 & Table S4. Median relative position per fragment type. & S-4 \\
\hline 5 & $\begin{array}{l}\text { Table S5. Area under the curve (AUC) values for receiver operating characteristic curves } \\
\text { generated using logistic regression model } 1 \text { and model } 2 \text { for each fragment type. }\end{array}$ & S-5 \\
\hline 6 & $\begin{array}{l}\text { Table S6. Fragment ion assignments in HCD and UVPD mass spectra for peptides AAAAA, } \\
\text { PAAAA, KAAAA, RAAAA. }\end{array}$ & S-6 \\
\hline 7 & $\begin{array}{l}\text { Figure S1. Estimated false discovery rate for fragment assignments in mass shifted fragment } \\
\text { lists for (A) ubiquitin, (B) myoglobin, and (C) bovine carbonic anhydrase based on UVPD. }\end{array}$ & S-7 \\
\hline 8 & $\begin{array}{l}\text { Figure S2. Percentage of c fragment assignments plotted as a function of (A) protein alpha- } \\
\text { helical content and (B) protein beta-sheet content. }\end{array}$ & S-8 \\
\hline 6 & Figure S3. Histogram of backbone cleavages for $y-1$ and $Y$ fragments. & S-9 \\
\hline 7 & Figure S4. Histogram of backbone cleavages for $a / x$ and $a+1 / x+1$ fragments. & S-10 \\
\hline 8 & Figure S5. Backbone cleavage intensities adjacent to each amino acid. & S-11 \\
\hline 9 & 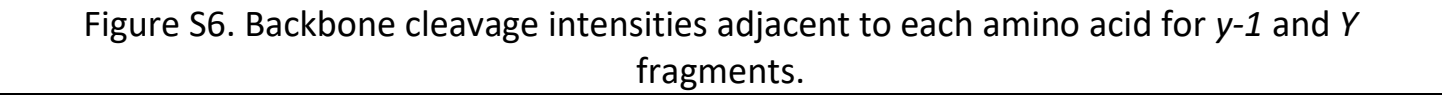 & S-12 \\
\hline 10 & $\begin{array}{l}\text { Figure S7. Intensity traces of sum intensity of fragment ions at each residue position } \\
\text { produced by top-down UVPD of ubiquitin }\end{array}$ & S-13 \\
\hline 10 & Figure S8. ERMS and $E_{50}$ values for AAAAA, PAAAA, KAAAA, and RAAAA. & S-14 \\
\hline 11 & Figure S9. UVPD spectra for AAAAA, PAAAA, KAAAA, and RAAAA. & S-15 \\
\hline 12 & Figure S10. Reaction scheme for carbamylation of primary amines & S-16 \\
\hline 13 & Figure S11. Deconvoluted spectra for apo and holo calmodulin & S-17 \\
\hline
\end{tabular}


Table S2. Proteins and protein complexes included in the native top-down $193 \mathrm{~nm}$ UVPD dataset.

\begin{tabular}{|c|c|c|c|c|c|c|}
\hline Protein & Oligomer State & Ligands & Charge State & $\begin{array}{c}\text { Isoelectric } \\
\text { Point } \\
\end{array}$ & $\begin{array}{c}\text { Monomer } \\
\text { Molecular Weight }\end{array}$ & PDB \\
\hline Adenylate Kinase & Monomer & Apo & 9 & 8.68 & $21.6 \mathrm{kDa}$ & - \\
\hline Adenylate Kinase & Monomer & Ap4a & 9 & 8.68 & $21.6 \mathrm{kDa}$ & - \\
\hline Alcohol Dehydrogenase & Homotetramer & Zinc & 25 & 6.21 & $36.7 \mathrm{kDa}$ & $7 \mathrm{KC} 2$ \\
\hline Aldolase & Homotetramer & Apo & 25 & 8.40 & $39.6 \mathrm{kDa}$ & 6ALD \\
\hline Alpha-Casein & Monomer & Copper & 9 & 4.91 & $23.6 \mathrm{kDa}$ & - \\
\hline Alpha-Synuclein & Monomer & Apo & 7 & 4.67 & $14.5 \mathrm{kDa}$ & - \\
\hline Aquaporin Z & Homotetramer & Apo & 14 & 6.50 & $24.7 \mathrm{kDa}$ & $2 A B M$ \\
\hline Barnase & Monomer & Apo & 7 & 8.16 & $14.3 \mathrm{kDa}$ & $1 \mathrm{~A} 2 \mathrm{P}$ \\
\hline Barnase & Heterodimer(Barnase $\bullet$ Barstar) & Apo & 10 & 8.16 & $14.3 \mathrm{kDa}$ & $1 \mathrm{X} 1 \mathrm{U}$ \\
\hline Barstar & Monomer & Apo & 6 & 5.39 & $12.1 \mathrm{kDa}$ & 1BTA \\
\hline Barstar & Heterodimer(Barnase $\bullet$ Barstar) & Apo & 10 & 5.39 & $12.1 \mathrm{kDa}$ & $1 X 1 U$ \\
\hline Beta-Casein & Monomer & Copper & 10 & 5.13 & $24.0 \mathrm{kDa}$ & - \\
\hline Beta-Lactoglobulin & Monomer & Apo & 8 & 4.76 & $18.4 \mathrm{kDa}$ & - \\
\hline Beta-Lactoglobulin & Homodimer & Apo & 12 & 4.76 & $18.4 \mathrm{kDa}$ & 1BEB \\
\hline Calmodulin & Monomer & Apo & 7 & 4.09 & $16.7 \mathrm{kDa}$ & 1CFD \\
\hline Calmodulin & Monomer & Calcium & 7 & 4.09 & $16.7 \mathrm{kDa}$ & $1 \mathrm{CLL}$ \\
\hline Carbonic Anhydrase I (Human) & Monomer & $\mathrm{Zn}$ & 10 & 6.63 & $28.8 \mathrm{kDa}$ & $610 \mathrm{~J}$ \\
\hline Carbonic Anhydrase I (Human) & Monomer & $\mathrm{Zn}$ & 9 & 6.63 & $28.8 \mathrm{kDa}$ & $610 \mathrm{~J}$ \\
\hline Carbonic Anhydrase II (Bovine) & Monomer & $\mathrm{Zn}$ & 10 & 6.40 & $29.0 \mathrm{kDa}$ & 1V9E \\
\hline Carbonic Anhydrase II (Bovine) & Monomer & $\mathrm{Zn}$ & 9 & 6.40 & $29.0 \mathrm{kDa}$ & $1 \mathrm{~V} 9 \mathrm{E}$ \\
\hline Carbonic Anhydrase II (Human) & Monomer & $\mathrm{Zn}$ & 10 & 6.86 & $29.1 \mathrm{kDa}$ & $3 \mathrm{KS} 3$ \\
\hline Carbonic Anhydrase II (Human) & Monomer & $\mathrm{Zn}$ & 9 & 6.86 & $29.1 \mathrm{kDa}$ & $3 \mathrm{KS} 3$ \\
\hline Concanavalin A & Homotetramer & Calcium, Manganese & 19 & 5.27 & $25.6 \mathrm{kDa}$ & $1 J B C$ \\
\hline Concanavalin A & Homodimer & Calcium, Manganese & 15 & 5.27 & $25.6 \mathrm{kDa}$ & - \\
\hline C-Reactive Protein & Homopentamer & Calcium & 24 & 5.28 & $23.0 \mathrm{kDa}$ & $1 \mathrm{GNH}$ \\
\hline Cytochrome C & Monomer & Heme & 7 & 9.59 & $12.3 \mathrm{kDa}$ & 1HRC \\
\hline E. coli ammonia channel & Homotrimer & Apo & 16 & 6.25 & $42.2 \mathrm{kDa}$ & $1 U 7 G$ \\
\hline Hemoglobin Subunit $\alpha$ & Heterotetramer $(2 \alpha \bullet 2 \beta)$ & Heme & 16 & 8.73 & $15.1 \mathrm{kDa}$ & 2DN2 \\
\hline Hemoglobin Subunit $\beta$ & Heterotetramer $(2 \alpha \bullet 2 \beta)$ & Heme & 16 & 6.81 & $15.9 \mathrm{kDa}$ & 2DN2 \\
\hline High-mobility group AT-hook 2 & Monomer & Apo & 7 & 10.81 & $11.6 \mathrm{kDa}$ & - \\
\hline Mechanosensitive Channel of Large Conductance & Homopentamer & Apo & 13 & 5.77 & $17.1 \mathrm{kDa}$ & 2OAR \\
\hline Myoglobin & Monomer & Heme & 8 & 7.36 & $16.9 \mathrm{kDa}$ & 1DWR \\
\hline Staph Nuclease & Monomer & Apo & 8 & 9.24 & $16.1 \mathrm{kDa}$ & 1STN \\
\hline Superoxide Dismutase & Homodimer & Copper, Zinc & 11 & 5.86 & $15.6 \mathrm{kDa}$ & $1 \mathrm{CBJ}$ \\
\hline Transthyretin & Monomer & Apo & 7 & 5.31 & $13.8 \mathrm{kDa}$ & - \\
\hline Transthyretin & Tetramer & Apo & 14 & 5.31 & $13.8 \mathrm{kDa}$ & 4TLT \\
\hline Trypsinogen & Monomer & Calcium & 9 & 8.23 & $24.0 \mathrm{kDa}$ & - \\
\hline Ubiquitin & Monomer & Apo & 5 & 6.56 & $8.6 \mathrm{kDa}$ & $1 U B O$ \\
\hline
\end{tabular}


Table S3. Number of assigned fragment ions based on type.

\begin{tabular}{|c|c|}
\hline $\begin{array}{c}\text { Fragment } \\
\text { Type }\end{array}$ & $\begin{array}{c}\text { Number of } \\
\text { Assignments }\end{array}$ \\
\hline$a$ & 1252 \\
\hline$a+1$ & 1286 \\
\hline$b$ & 698 \\
\hline$c$ & 1043 \\
\hline$x$ & 998 \\
\hline$x+1$ & 1037 \\
\hline$y$ & 963 \\
\hline$y-1$ & 932 \\
\hline$Y$ & 658 \\
\hline$z$ & 787 \\
\hline
\end{tabular}


Table S4. Median relative position ${ }^{1}$ per fragment type.

\begin{tabular}{|c|c|}
\hline $\begin{array}{c}\text { Fragment } \\
\text { Type }\end{array}$ & $\begin{array}{c}\text { Median Relative } \\
\text { Position }\end{array}$ \\
\hline$a, a+1$ & 0.24 \\
\hline$x, x+1$ & 0.75 \\
\hline$a$ & 0.27 \\
\hline$a+1$ & 0.22 \\
\hline$x$ & 0.73 \\
\hline$x+1$ & 0.76 \\
\hline$b$ & 0.16 \\
\hline$y$ & 0.86 \\
\hline$c$ & 0.19 \\
\hline$z$ & 0.82 \\
\hline$y-1$ & 0.72 \\
\hline$y$ & 0.73 \\
\hline
\end{tabular}

${ }^{1}$ The median relative position refers to the median value obtained from the distribution of backbone cleavage positions where 0 represents the $\mathrm{N}$-terminus and 1.00 represents the $\mathrm{C}$-terminus. 
Table S5. Area under the curve (AUC) values for receiver operating characteristic curves generated using logistic regression model 1 and model 2 (Eq. 2 and Eq. 3) for each fragment type.

\begin{tabular}{|c|c|c|}
\hline & Model1 AUC & Model2 AUC \\
\hline $\mathbf{a}$ & 0.84 & 0.85 \\
\hline $\mathbf{a + 1}$ & 0.88 & 0.89 \\
\hline $\mathbf{x}$ & 0.80 & 0.85 \\
\hline $\mathbf{x + 1}$ & 0.82 & 0.86 \\
\hline $\mathbf{b}$ & 0.87 & 0.87 \\
\hline $\mathbf{y}$ & 0.87 & 0.88 \\
\hline $\mathbf{C}$ & 0.88 & 0.88 \\
\hline $\mathbf{z}$ & 0.85 & 0.88 \\
\hline $\mathbf{y}-\mathbf{1}$ & 0.80 & 0.85 \\
\hline $\mathbf{Y}$ & 0.84 & 0.86 \\
\hline
\end{tabular}


Table S6. Fragment ion assignments in HCD and UVPD mass spectra for peptides AAAAA, PAAAA, KAAAA, RAAAA. Internal fragments are denoted by two fragment assignments separated by a dash "-".

\begin{tabular}{|c|c|c|c|}
\hline & HCD ions & UVPD: HCD-like ions & UVPD: Unique to UVPD \\
\hline AAAAA & $\begin{array}{c}\mathrm{b} 3, \mathrm{~b} 2, \mathrm{~b} 4, \mathrm{y} 3, \mathrm{y} 2, \mathrm{a} 4, \mathrm{a} 3-\mathrm{NH} 3, \\
\mathrm{y} 4, \mathrm{a} 4-\mathrm{NH} 3, \mathrm{a} 2, \mathrm{M}-\mathrm{CO} 2, \mathrm{M}- \\
\mathrm{H} 2 \mathrm{O}\end{array}$ & $\begin{array}{c}\mathrm{b} 3, \mathrm{~b} 2, \mathrm{~b} 4, \mathrm{y} 3, \mathrm{y} 2, \mathrm{a} 4, \mathrm{a} 3-\mathrm{NH} 3, \\
\mathrm{y} 4, \mathrm{a} 4-\mathrm{NH} 3, \mathrm{a} 2\end{array}$ & $\begin{array}{c}\mathrm{c} 4-\mathrm{CH} 4, \mathrm{a} 4+1, \mathrm{a} 3+1, \mathrm{Y} 2, \\
\text { z2-CO2, M-COOH. }\end{array}$ \\
\hline PAAAA & $\begin{array}{c}\text { b3, b2, b4, a2, a4, a1, a3, y3, } \\
\text { y2, y4, y4-b4, y4-a3 }\end{array}$ & $\begin{array}{c}\mathrm{b} 3, \mathrm{~b} 2, \mathrm{~b} 4, \mathrm{a} 2, \mathrm{a} 4, \mathrm{a} 1, \mathrm{a} 3, \mathrm{y} 3, \mathrm{y} 2 \\
\mathrm{y} 4, \mathrm{y} 4-\mathrm{b} 4, \mathrm{y} 4-\mathrm{a} 3\end{array}$ & $\begin{array}{c}\mathrm{a} 3+1, \mathrm{a} 2+1, \mathrm{c} 1, \mathrm{c} 3+1, \mathrm{M}- \\
\mathrm{COOH} \cdot\end{array}$ \\
\hline KAAAA & $\begin{array}{c}\text { b4, b3, b2, b1, a1, y2, y3, a2, } \\
\text { a3, a4, y4, b2-NH3, b3-NH3, } \\
\text { y4-b4, y4-a3, y4-b3 }\end{array}$ & $\begin{array}{c}\text { b4, b3, b2, b1, a1, y2, y3, a2, a3, } \\
\text { a4, y4, b2-NH3, b3-NH3, y4-b4, } \\
\text { y4-a3, y4-b3 }\end{array}$ & $\begin{array}{c}\mathrm{a} 4+1, \mathrm{a} 3+1, \mathrm{a} 2+1, \mathrm{M}- \\
\mathrm{COOH} \cdot\end{array}$ \\
\hline RAAAA & $\begin{array}{c}\text { a2-NH3, b2-NH3, M-NH3, a 1- } \\
\text { NH3, y4-a3, b1-NH3, a2- } \\
\text { guanine, y4-b3, b1, y2, b2- } \\
\text { guanine, a2, y4-b4, y3, b3- } \\
\text { guanine, a3-NH3, a3, b3-NH3, } \\
\text { b3, y4, b4-guanine, a4-NH3, } \\
\text { a4, b4 }\end{array}$ & $\begin{array}{c}\text { a2-NH3, b2-NH3, M-NH3, a 1- } \\
\text { NH3, y4-a3, b1-NH3, a2-guanine, } \\
\text { y4-b3, b1, y2, a2, b3-guanine, a3, } \\
\text { b3, a4, b4 }\end{array}$ & $\begin{array}{c}\mathrm{a} 4+1, \mathrm{c} 3, \mathrm{a} 3+1, \mathrm{c} 2, \mathrm{a} 2+1, \\
\mathrm{c} 1, \mathrm{M}-\mathrm{COOH} \cdot\end{array}$ \\
\hline
\end{tabular}



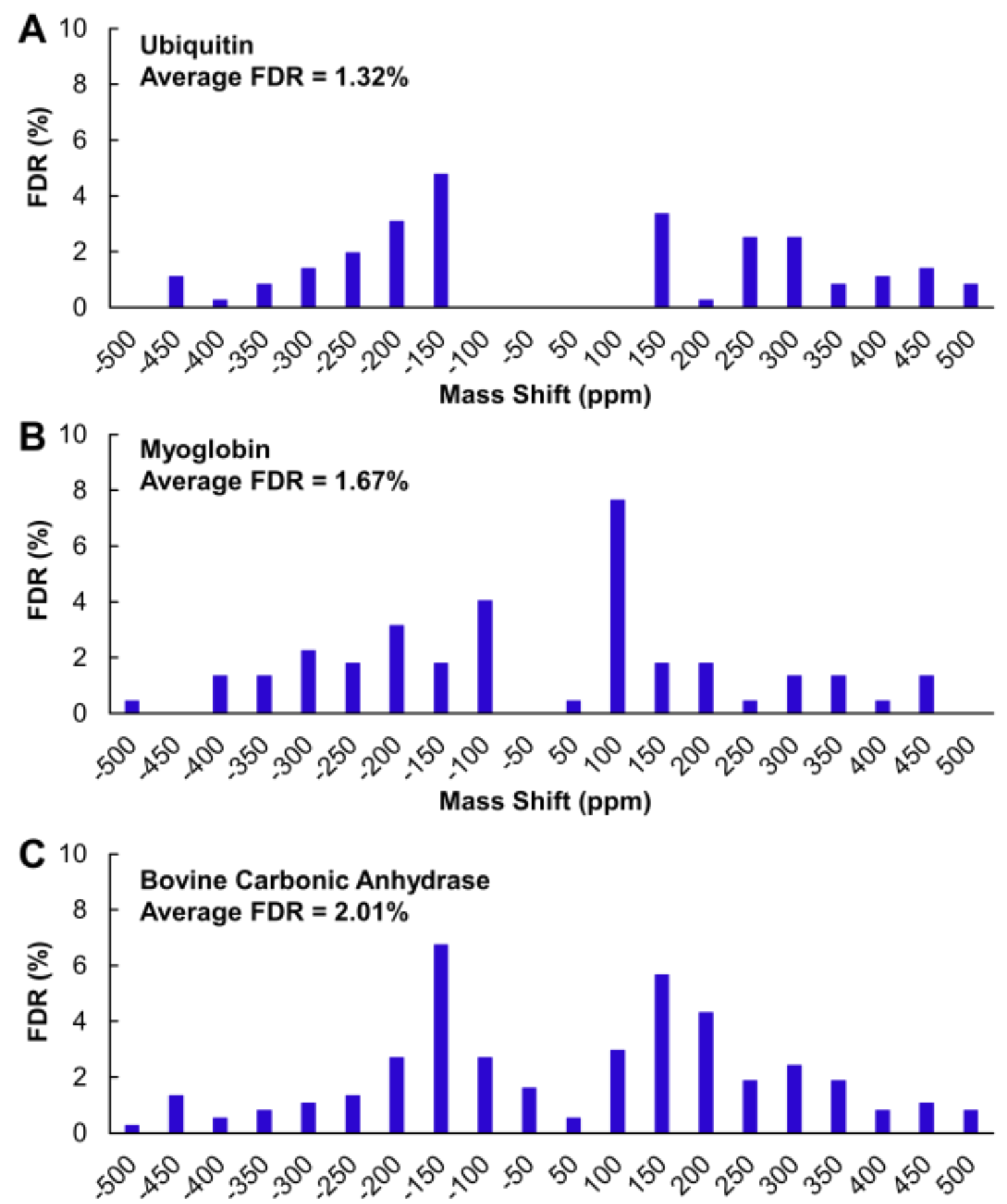

Mass Shift (ppm)

Figure S1. Estimated false discovery rate for fragment assignments in mass shifted fragment lists for (A) ubiquitin, (B) myoglobin, and (C) bovine carbonic anhydrase based on UVPD. 

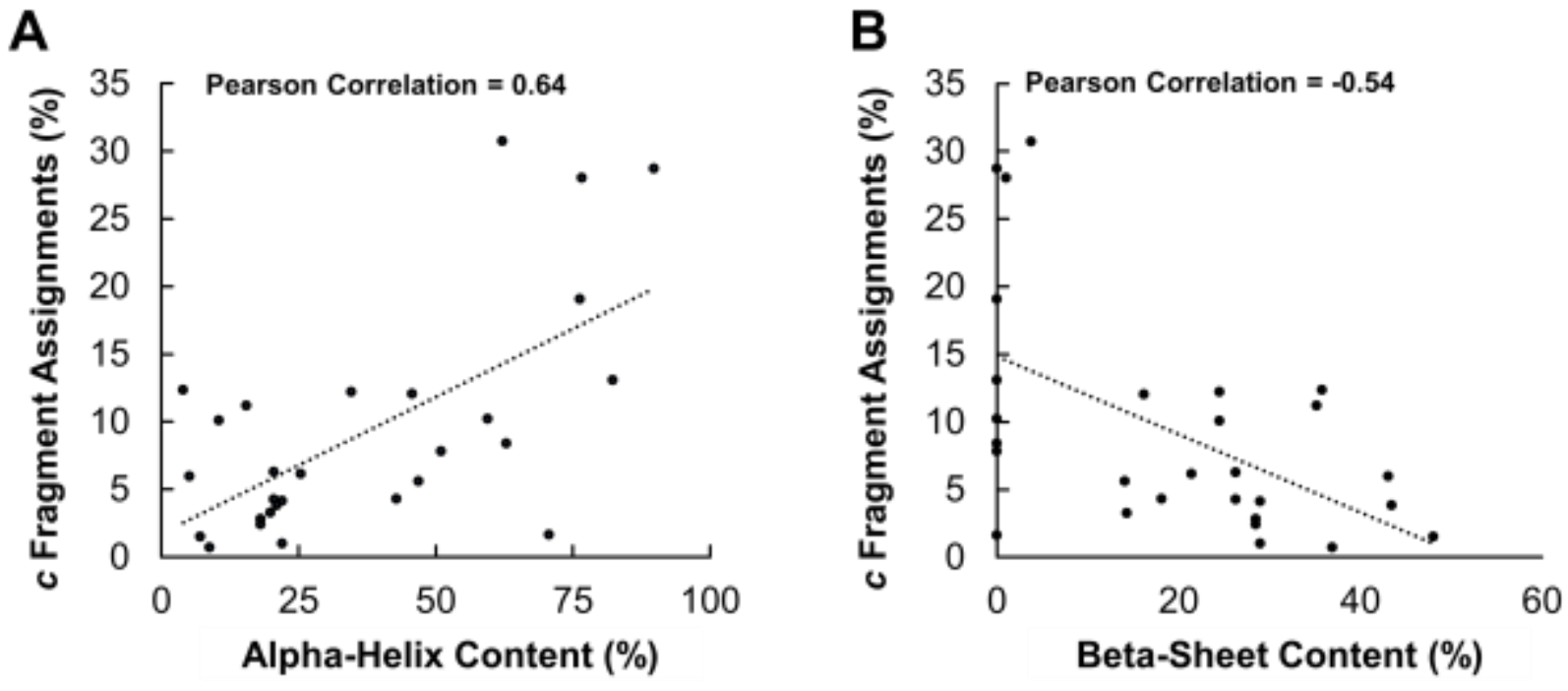

Figure S2. Percentage of $c$ fragment assignments plotted as a function of (A) protein alpha-helical content and (B) protein beta-sheet content. 

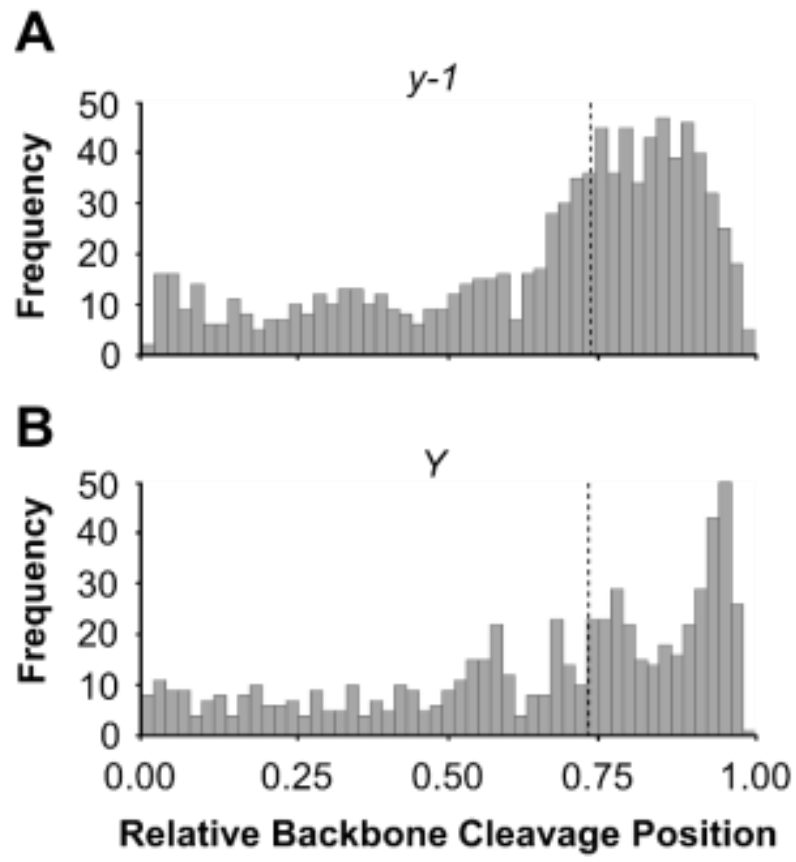

Figure S3. (A) Histogram of backbone $y-1$ cleavages (total of 932 fragments) along the protein sequence (divided into 50 bins). The relative backbone position extends from the $\mathrm{N}$-terminus $(0.00)$ to the $\mathrm{C}$-terminus (1.00). (B) Histogram of backbone $Y$ cleavages (total of 658 fragments) along the protein sequence (divided into 50 bins). The median relative position for each fragment type is demarcated by a vertical dashed grey line. 
A

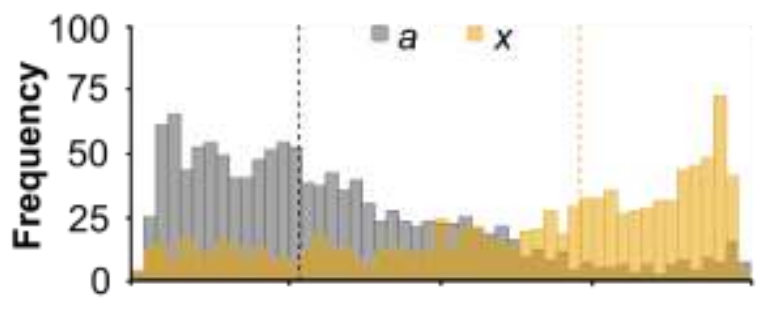

B

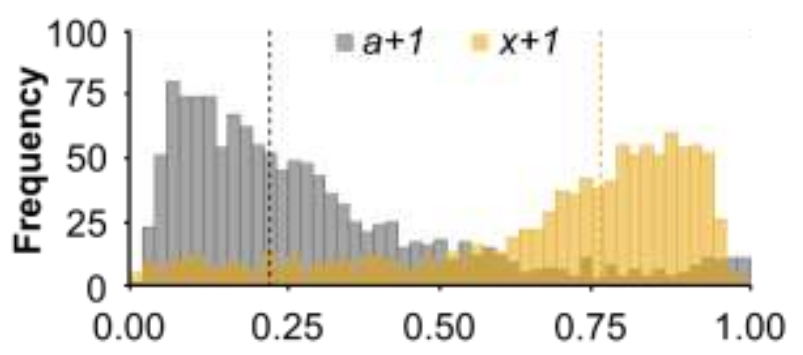

Relative Backbone Cleavage Position

Figure S4. (A) Histogram of backbone $a$ and $x$ cleavages (total of 1252 and 998 fragments, respectively) along the protein sequence (divided into 50 bins). The relative backbone position extends from the $\mathrm{N}$ terminus (0.00) to the C-terminus (1.00). (B) Histogram of backbone $a+1$ and $x+1$ cleavages (total of 1286 and 1037 fragments, respectively) along the protein sequence (divided into 50 bins). The median relative position for N-terminal containing and C-terminal containing fragment ions are demarcated by a vertical dashed grey or gold line, respectively. 


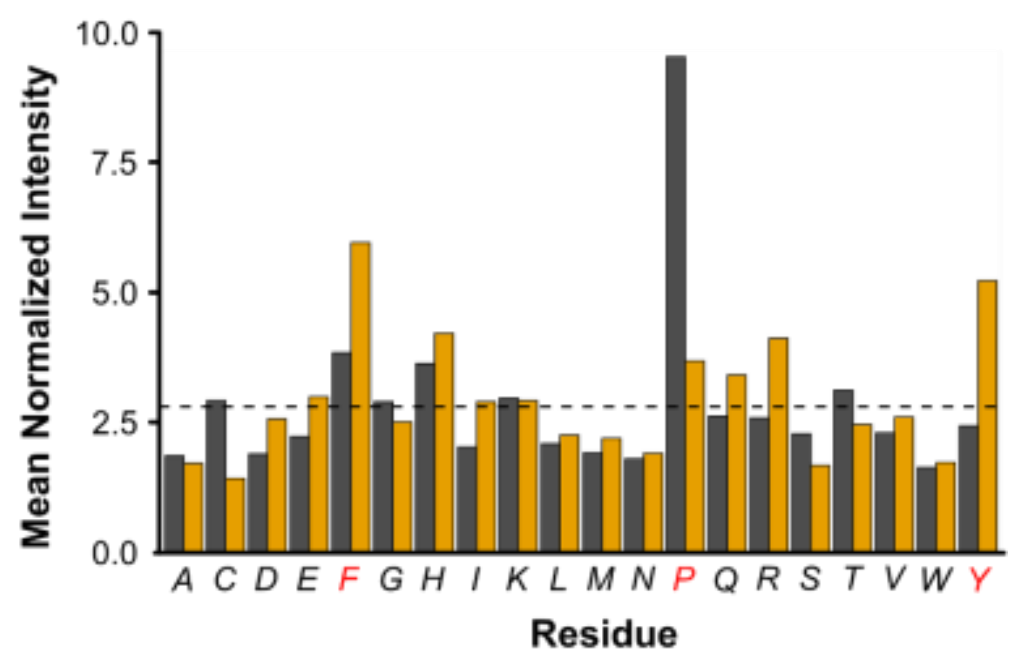

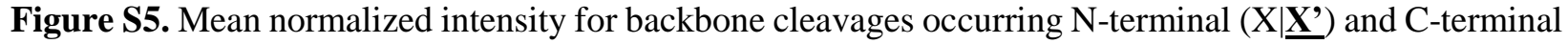
$\left(\underline{\mathbf{X}} \mid \mathrm{X}^{\prime}\right)$ to each amino acid are displayed as grey bars and gold bars, respectively, considering all fragment types $(a, a+1, b, c, x, x+1, y, y-1, Y, z)$. Mean fragment intensity that would be achieved if fragment abundances were evenly distributed across each amino acid is shown as a horizontal dashed line. This histogram is based on a total of 9,654 fragment ions. 

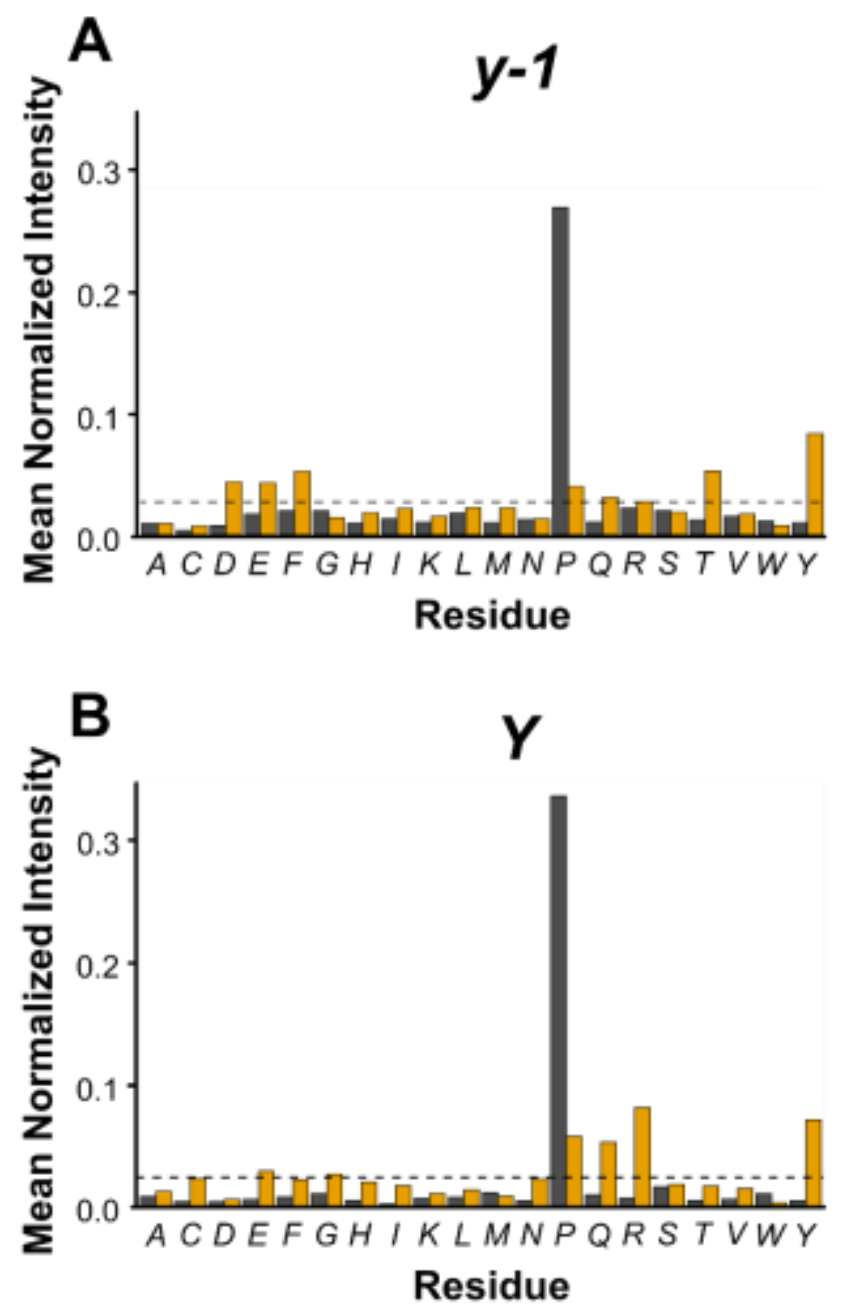

Figure S6. Mean normalized intensity for fragment ions originating from backbone cleavages occurring Nterminal (X|X ' gray bars, fragment ions retaining the C-terminus) and C-terminal ( $\underline{\mathbf{X}} \mid \mathrm{X}^{\prime}$ gold bars, fragment ions retaining the $\mathrm{N}$-terminus) to each amino acid are displayed as grey bars and gold bars, respectively for (A) $y-1$ and (B) $Y$ fragment types. Mean fragment intensity that would be achieved if fragment abundances were uniformly distributed at every backbone position is shown as a horizontal dashed line. The histograms are based on $932 y-1$ ions and $658 Y$ ions. 


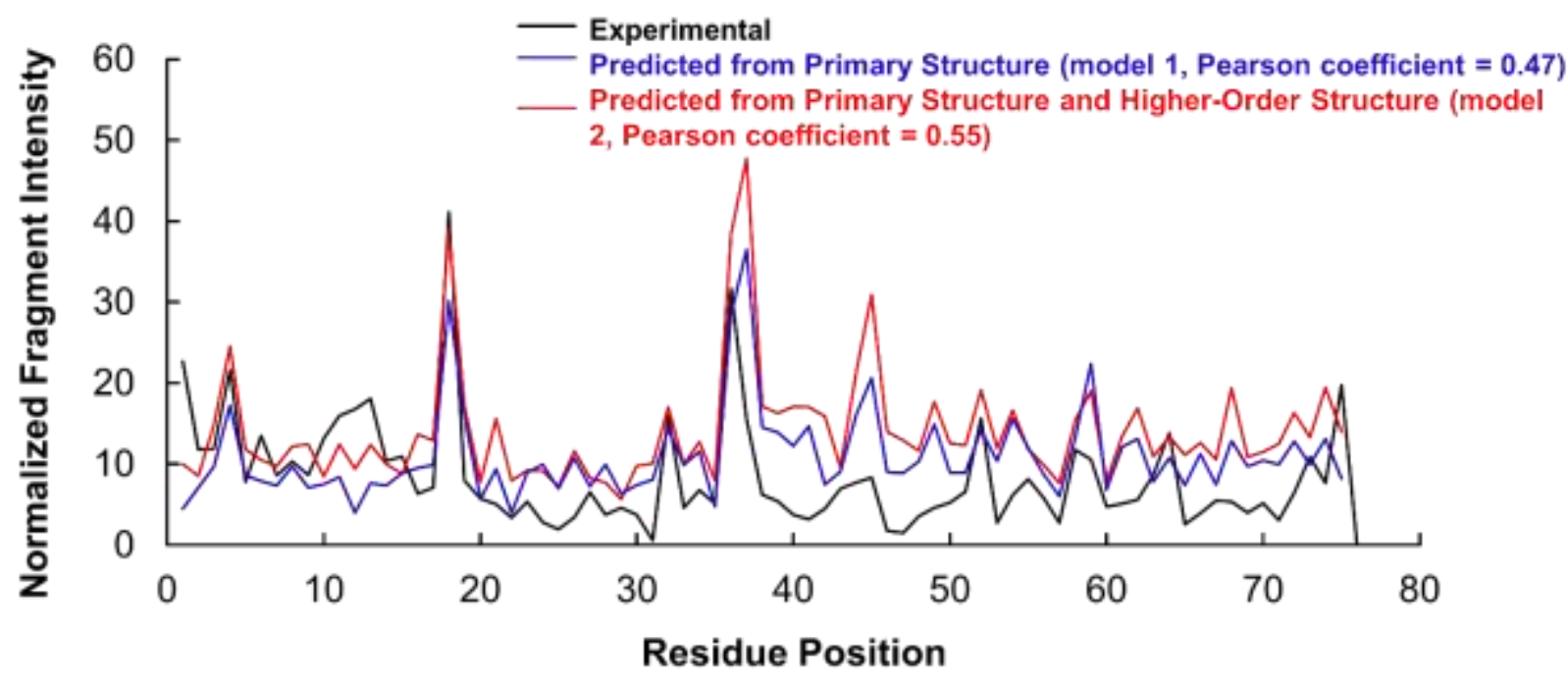

Figure S7. Intensity traces of sum intensity of fragment ions originating from backbone cleavages at each residue position produced by UVPD of ubiquitin (black trace), predicted using primary structure of ubiquitin (blue trace) or predicted using primary structure and higher order structure of ubiquitin (red trace). Pearson correlation coefficient between the experimental trace and model 1 trace is 0.47. Pearson correlation coefficient between the experimental trace and model 2 trace is 0.47 . 

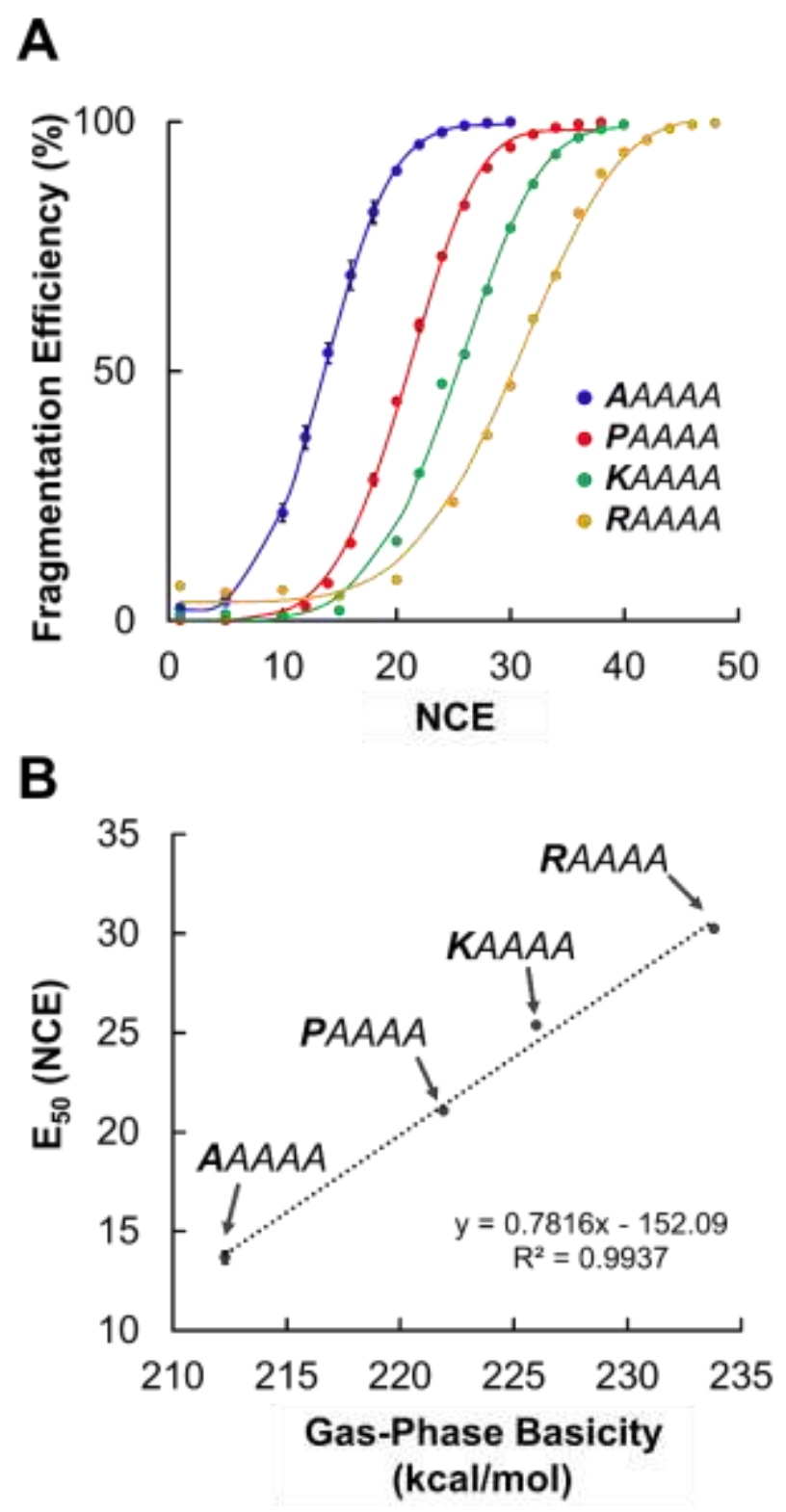

Figure S8. (A) Fragmentation efficiency curves generated by $\mathrm{HCD}$ for the $[\mathrm{M}+\mathrm{H}]$ ion of AAAAA, PAAAA, KAAAA, RAAAA. The y-axis shows fragmentation efficiency (sum fragment ion intensity divided by sum of all ion intensities) at each normalized collision energy (NCE). (B) Inflection points ( $\left.\mathrm{E}_{50}\right)$ for each peptide in (A) was plotted against the gas phase basicity of the N-terminal amino acid from reference 57. 


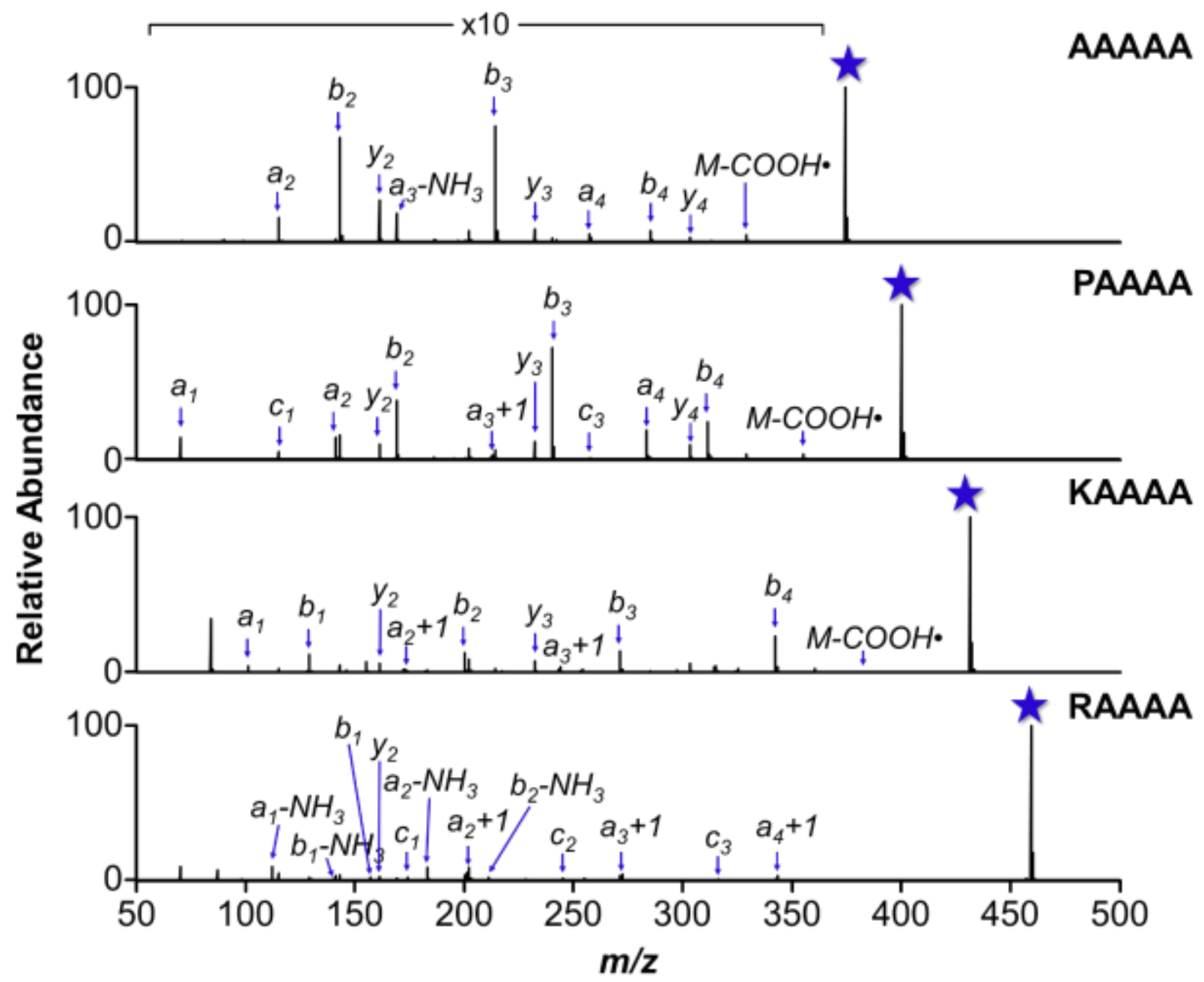

Figure S9. UVPD (1 pulse at $3 \mathrm{~mJ}$ ) mass spectra of single protonated AAAAA, PAAAA, KAAAA, and RAAAA. Precursor ions are denoted by a star. 


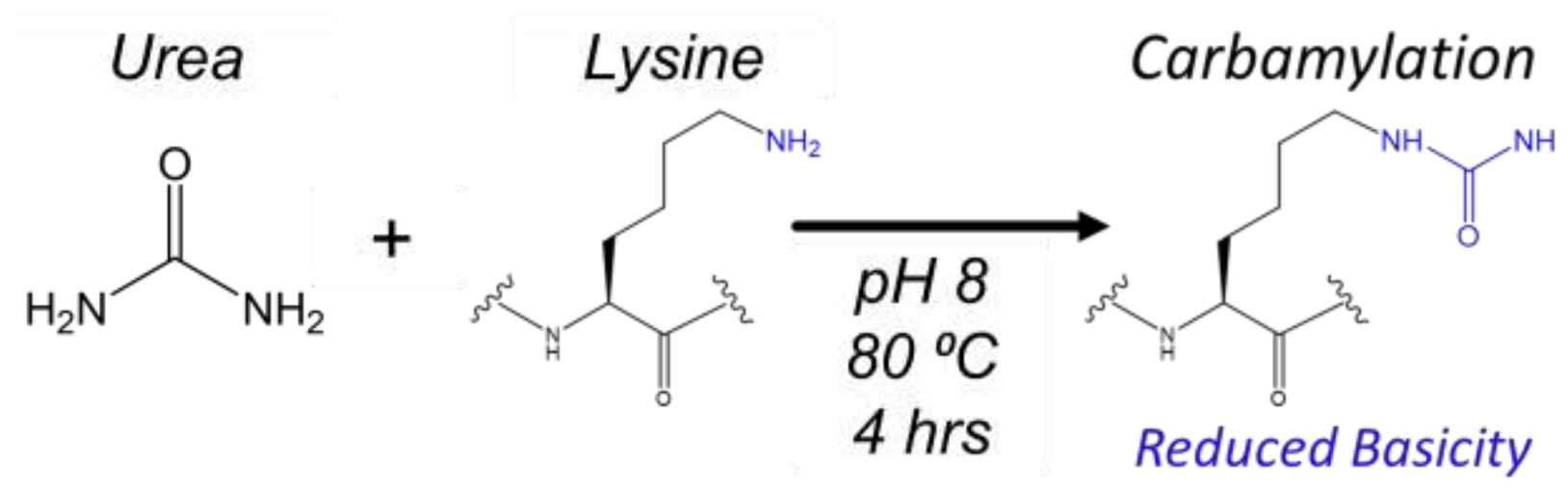

Figure S10. Reaction scheme for carbamylation of primary amines. 

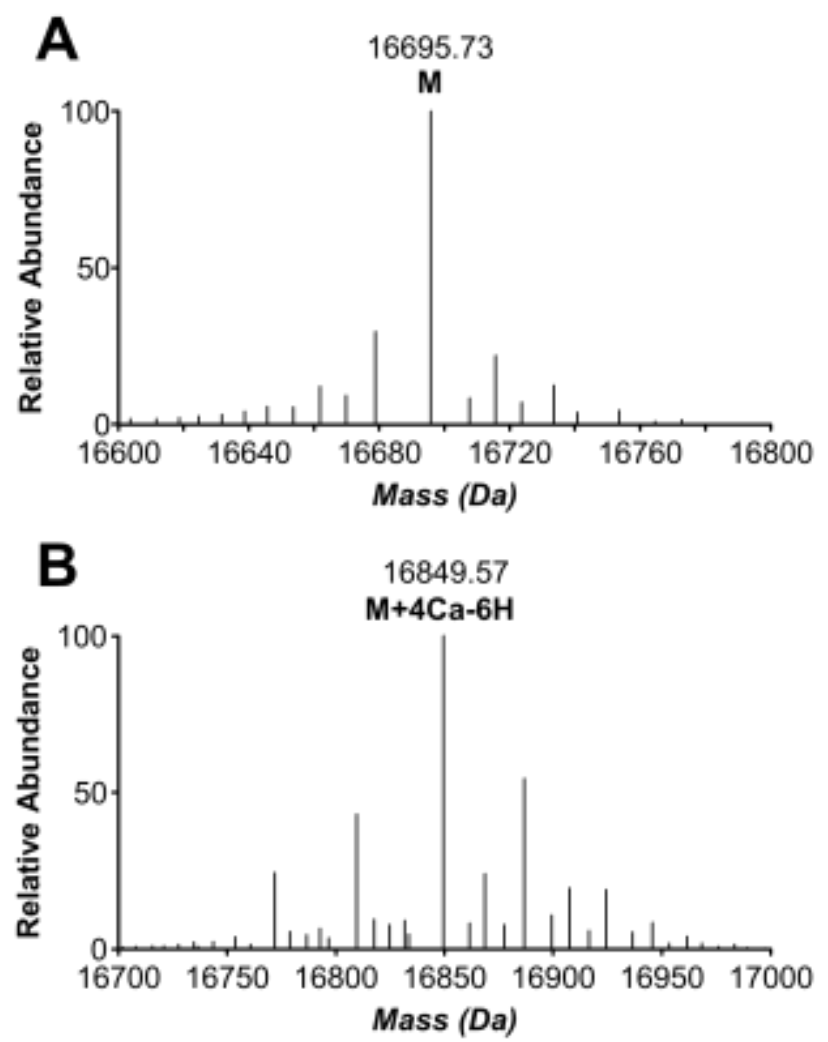

Figure S11. Deconvoluted MS1 spectra for the 7+ charge state of (A) apo-calmodulin and (B) calciumbound calmodulin (calmodulin $\bullet$ Ca). 